\title{
The decomposition of visual binding over time: Neuropsychological evidence from illusory conjunctions after posterior parietal damage
}

Running head: Decomposing visual binding over time

\author{
Céline R. Gillebert ${ }^{1,2}$ and Glyn W. Humphreys ${ }^{3}$ \\ ${ }^{1}$ Laboratory of Experimental Psychology, University of Leuven (K.U.Leuven), Belgium \\ ${ }^{2}$ Laboratory for Cognitive Neurology, University of Leuven (K.U.Leuven), Belgium \\ ${ }^{3}$ School of Psychology, University of Birmingham, UK
}

Address correspondence to Céline R. Gillebert (celine.gillebert@ med.kuleuven.be) and Glyn W. Humphreys (g.w.humphreys@bham.ac.uk).

This is a pre-copy-editing, author-produced PDF of an article accepted for publication in Visual Cognition following peer review. The definitive publisher-authenticated version (Gillebert, C.R., and Humphreys, G.W. (2008). The decomposition of visual binding over time: Neuropsychological evidence from illusory conjunctions after posterior parietal damage. Visual Cognition, 18:954-980) is available online at: http://dx.doi.org/10.1080/13506280903356764. 
Decomposing visual binding over time

\section{ABSTRACT}

Patients with Bálint`s syndrome are known to make abnormal numbers of illusory conjunctions (ICs) when presented with multiple stimuli and asked to report the features of one. We used two converging procedures to assess the time course of these errors. In Experiments 1 and 2 the errors produced by a patient with Bálint`s syndrome, GK, were examined as a function of when he responded. We find that ICs were present even in GK's fastest responses, but that they also increased when GK responded slowly. In Experiment 3 we varied the exposure duration of the stimuli. With short stimulus exposures GK made ICs that he was certain were correct. With longer exposures there was an increase in the number of ICs where GK expressed uncertainty. In contrast to these 'uncertain' ICs, feature errors decreased as the exposure duration increased. We propose that the ICs present in GK's fastest responses, and that arise with short stimulus exposures, reflect impairments at a first stage of binding. In addition to this, 'uncertain' ICs arise on trials with slow responses, and with long exposures, due to performance then being affected by impairments to a second process dependent on bound features being consolidated into a more stable representation. The role of this consolidation process is limited when responses are made rapidly and exposure durations limited. This two-stage account is discussed in relation to other accounts of feature binding. 
Decomposing visual binding over time

\section{ACKNOWLEDGEMENTS}

This work was supported by grants from the BBSRC, the EU, the MRC and the Stroke Association (UK). C.R.G. is a research fellow of the Research Foundation - Flanders (FWO),

Belgium. We are grateful for the valuable comments made by Liqiang Huang, Joshua Cosman and one anonymous reviewer, which helped us to strengthen this article. 


\section{INTRODUCTION}

The outer world appears to us as a coherent whole, containing unified static or moving objects characterised by a certain shape, a certain colour, certain surface properties, and so forth. However, there is considerable evidence from neurophysiology and neuropsychology that the basic visual features of objects are processed quasi-independently in specialised cortical areas during the early stages of visual perception (Hubel \& Livingstone, 1987; see chapters 1 and 2 in Humphreys, 1999). This raises the question of how unified perception occurs, given the wide cortical distributions of visual features, the large receptive fields of neurons in at least some parts of the cortical visual stream (e.g., inferior temporal cortex), and the separation of different visual pathways. In other words, how do features become 'bound together' to generate coherent perceptual and memory representations of the world? In the present study we assess the processes involved in feature binding through a neuropsychological analysis of a patient with Bálint`s syndrome.

One way to gain insights into the processes underlying binding in vision and memory is to examine how the binding process breaks down under particular conditions. Treisman and Schmidt (1982) first reported that, when normal participants are prevented from attending fully to visual stimuli, they can make so-called 'illusory conjunction errors' (ICs), where they miscombine features belonging to different objects. The rate of ICs can be greater than the rate of mis-reports of features not present in displays ('feature errors', FEs), and they can occur even when participants only have to verify that a particular combination of features is present. Treisman and Schmidt (1982) argued that, when forced-choice verification is required, ICs due to forgetting the combination of features during perceptual report should be minimal. They concluded that ICs reflect errors in perceptual coding or encoding into visual short-term memory 
(VSTM) rather than participants forgetting the relations between conjunctions of features in memory (though see Donk, 2001, for an alternative view).

Following damage to posterior parietal cortex, patients can make binding errors in perception even when they are given time to attend fully to stimuli (Cohen \& Ivry, 1989; Cohen \& Rafal, 1991; Friedman-Hill, Robertson, \& Treisman, 1995; Humphreys, Cinel, Wolfe, Olson, \& Klempen, 2000). Such patients typically have problems in the control of visual attention to displays (see Friedman-Hill et al., 1995; Humphreys et al., 2000, for evidence) and they also have poor spatial localisation (Humphreys \& Riddoch, 2003). The abnormal numbers of ICs generated by such patients could thus reflect either the necessary role of visual attention in feature binding in perception (Treisman, 1998) or VSTM (Luck \& Vogel, 1997; Wheeler \& Treisman, 2002), or the need for accurate location coding to align stimuli coming from different stimulus domains (Cohen \& Ivry, 1989; Humphreys et al., 2000).

Interestingly, patients who make abnormal numbers of ICs when reporting the colours and shapes of stimuli do not necessarily make binding errors when dealing with visual forms. For example, the perceptual report of such patients can be strongly affected by form-based grouping between visual elements, sensitive to factors such as collinearity, closure, common form, common enclosure and even stored knowledge of particular forms (Humphreys, 1998; Humphreys et al., 2000; Kumada \& Humphreys, 2001; Ward, Goodrich, \& Driver, 1994). These data suggest that the processes leading to the binding of elements into integrated visual forms can be distinguished from the processes involved in binding together form and surface features, such as colour (Humphreys, 2001). That is, rather than there being a single binding operation in vision (e.g., Treisman, 1998), there may be several, some of which operate within the form domain and some of which operate across domains of feature representations (e.g., between 
colour and form). The evidence suggests that the posterior parietal cortex may be required particularly for binding features across different domains, possibly because location codes from different domains are combined within this region of cortex (Robertson, 2003), with the posterior parietal cortex providing 'a master map' of locations (see Treisman, 1998). Evidence for this argument comes from recent work with unilateral parietal patients where the difficulty of detecting cross-domain conjunctions was matched to that of detecting low saliency features and form conjunctions, when stimuli appeared in the patients' ipsilesional fields. Despite this, the parietal patients remained selectively impaired at discriminating cross-domain conjunctions in their contralesional field (Humphreys, Hodsoll \& Riddoch, 2009).

Although patients with damage to posterior parietal cortex are poor at explicitly reporting conjunctive relations between visual features, there is also evidence for 'implicit' binding between the same features. Effects of 'implicit' binding are found when responses to other stimuli are influenced by the conjunctive coding, even though patients fail to explicitly report the relations between the critical features. For example, Cinel and Humphreys (2006), Robertson, Treisman, Friedman-Hill and Grabowecky (1997) and Wocjiulik and Kanwisher (1998) have all reported that patients can show Stroop interference in colour naming (slower reaction times to name the colour blue when it forms the word RED than the word BLUE), despite the patients being unable to make judgements at a better than chance level of which colour was linked to which word. Cinel and Humphreys (2006) have further shown that these implicit effects are influenced by the same factors that influence explicit report of conjunctive information, including the spacing between the stimuli. These authors suggest that implicit binding effects are not qualitatively different from explicit binding (i.e., they are not based on functionally independent processes), but rather implicit binding effects are based on a rapid but unstable form 
of binding between visual features, which is usually later consolidated by slower, top-down reentrant activation from the posterior parietal lobe. This consolidation process leads to more stable representations of binding relations between stimuli, which are typically used as the basis for explicit report. If this re-entrant consolidation process is damaged, the patients make binding errors in explicit report, even if effects of the initial binding can be detected using implicit procedures. This proposal is consistent with the Reverse Hierarchy Theory (Hochstein \& Ahissar, 2002), and with Adaptive Resonance Theory (Grossberg, 1987), both of which propose that bottom-up processes first generate a coarse representation of stimuli, followed subsequently by confirmation or refutation of that representation by top-down re-entrant feedback.

Evidence for implicit binding is also of interest because it is difficult to attribute this to processes operating after a stable representation of the stimulus has been formed and coded in VSTM. It could be argued, for instance, that there is initially intact coding of conjunctive information into VSTM followed by rapid loss of the binding information. However, if there is spared coding into VSTM then it is difficult to see why effects only arise implicitly and patients cannot explicitly report the appropriate relationship - this is especially the case as the studies typically only require report of a single stimulus, minimising the VSTM load.

In the present paper we test the hypothesis that binding may indeed take place in at least two stages, with an initial, transitory coding of binding relationships followed by a later reentrant consolidation process. We report data from GK, a patient with bilateral posterior parietal damage, and we use two converging procedures to examine the time course of visual binding. In the first experiments (Experiments 1 and 2), we collected sufficient data to examine the time course of GK's errors. We assume that there is a monotonic relationship between the length of GK's responses and the time available for the different stages of visual processing, and that 
responses may be elicited from representations formed at each stage ${ }^{1}$. Fast responses may allow less time than slow responses for consolidation to play a role. Fast responses, then, may be more dependent on whether or not feature bindings are formed correctly at a first encoding stage, not whether consolidation is 'noisy' or not. Slow responses will allow more time for the consolidation process to influence performance, generally increasing the accuracy of responding. However, in a patient with impaired consolidation it is possible that the proportions of ICs will increase when there is more opportunity for a noisy consolidation process to influence performance. Hence ICs could show proportional increases as response times lengthen. Experiment 3 used a different approach. Here instead of using response times as a proxy measure of the length of processing time we directly manipulated the exposure duration of the displays. Short exposures may depend primarily on the probability of forming initial feature bindings. Long exposures should allow consolidation processes to affect performance. We again predict that, in a patient with impaired consolidation, ICs may increase proportionately at long relative to short stimulus exposures. This pattern has recently been reported in a patient with Bálint`s syndrome by Braet and Humphreys (2009). Here we replicate and extend this result with GK by having him rate the certainty of his responses. We report a difference in GK's perceptual certainty of ICs according to whether short or long exposures were used. When ICs were generated with short exposures, GK was often sure that his (erroneous) response was incorrect. We attribute these errors to incorrect initial perceptual binding. With long exposures, there was an increase in the proportion of ICs made, and, when these errors occurred, GK was very often

\footnotetext{
${ }^{1}$ This assumption distinguishes our proposal from ideas such as those put forward by Chun and Potter (1995) who argue that consolidation is necessary for any perceptual report. Instead of this we suggest that consolidation helps form a more stable representation, but that report can be based on non-consolidated as well as consolidated representations.
} 
uncertain of the response. We suggest that these 'uncertain ICs' reflect impaired re-entrant consolidation, which, in his case, adds noise to the initial encoding. We discuss the results in relation to different theories of visual feature binding.

\section{CASE REPORT}

GK (male, born in 1939, right-handed) suffered from two strokes in 1986, which resulted in damage to the right occipito-parietal, the right temporo-parietal and the left temporo-parietal areas. His brain damage lead to Bálint`s syndrome, including symptoms of simultanagnosia, optic ataxia and left-side extinction (Gilchrist, Humphreys, \& Riddoch, 1996; Humphreys et al., 2000). These symptoms made GK poor at the interpretation of complex scenes and gave rise to reading problems, as manifested in left-side neglect and attentional dyslexia. Despite these visual deficits, GK functioned at a high-level in many aspects of everyday life and had intact memory and comprehension.

\section{EXPERIMENT 1: The relation between ICs and response latencies}

In Experiment 1 we introduce the basic paradigm used in the paper along with our first exploration of the time course of ICs - in this case evaluating the production of ICs in relation to the speed of the response. GK was asked to report the colour and identity of a single letter at fixation. Simultaneous with the target letter another (distractor) letter could be presented in either his right or left visual field. Although the distractor was irrelevant to GK's task, he made IC errors in which he incorporated features from the distractor into his response to the target. It is these ICs that we focus on. We assessed (1) how the rate of ICs varied as a function of the time at which they were generated, in the distribution of reaction times (RTs) generated by GK across 
multiple trials, and (2) whether ICs represented features from stimuli in the left and right field equally, given that GK had a spatial bias favouring his right visual field (e.g., Gilchrist et al., 1996).

\section{METHOD}

We presented displays containing one or two letters, drawn in different colours. GK was asked to identify the letter and the colour of the stimulus at fixation and to ignore any distractors in the periphery. The letters and colours were randomly selected from a set of four values, respectively $\mathrm{A} / \mathrm{B} / \mathrm{C} / \mathrm{D}$ and blue/green/red/orange. Letters were drawn in upper case Arial Font and extended $3.2^{\circ}$ visual angle in height and in width. In each block, we presented 32 trials containing a single letter display and 48 containing a two-letter display, of which 24 with a distractor in the left visual field and 24 with a distractor in the right visual field. In two-letter displays, target and distractor differed both in letter and in colour and were separated by a horizontal gap of $7^{\circ}$ visual angle. A trial began with a central fixation cross and GK reported when he fixated on the cross. Once fixated, a display containing one or two letters was exposed for $3000 \mathrm{~ms}$ (see Figure 1a). GK was asked to identify the central target as soon as possible and the experimenter keyed the response into the computer. The experimenter was blind to the experimental condition. This procedure was used because GK found it difficult to make key presses himself. However, as his RTs were relatively slow, any timing errors can be considered negligible. We ran 25 blocks of trials, leading to 800 single item trials, 600 trials with a right distractor and 600 trials with a left distractor. In addition, we ran an additional four blocks of trials in which GK made key presses himself.

(insert Figure 1 about here) 


\section{RESULTS}

\section{Errors}

A variety of errors were made in this task (see also Figure 1b). Illusory conjunctions (ICs) arose when GK identified the features in the display but erroneously combined the colours and the shapes. This would be reflected in the correct report of one target feature, along with the report of one distractor feature. Feature errors (FEs) occurred when the patient reported one correct target feature and one feature not presented in the display. A third type of error was made when the patient correctly reported the distractor presented in the periphery instead of the target at fixation. This represents a form of extinction error, where the distractor appeared to extinguish the target. 'Other' errors included failures to report any item and the report of one distractor feature along with one feature not presented in the display.

\section{Overall performance}

GK correctly identified the target on $88 \%$ of the single letter trials. Errors included misreports of the letter identity (25), the colour (67) or both (5). On two-letter trials, overall performance was significantly better on trials with a left distractor $(85 \%$, close to performance on single letter trials) than on trials with a right distractor $(33 \%)\left(\chi^{2}(1)=329.13, p<.0001\right)$. Thirty-five out of the 2000 trials were removed from further analyses, because the RTs during those trials were not correctly keyed due to GK stuttering or making a cough.

\section{ICs versus FEs}

Following other authors (e.g., Cohen \& Rafal, 1991), we assessed the rate of ICs relative to the 
rate of FEs. Given the correct report of one, but not both, target features, we would expect $33 \%$ of the errors to be ICs and $67 \%$ of the errors to be FEs by chance. The observed proportion of ICs in GK's errors was larger than expected by chance, both for trials with a left distractor $(z=$ $3.80, p<.0001)$ and for trials with a right distractor $(z=13.80, p<.0001)$. However, the relative rate of ICs was larger on trials with a right distractor (120 ICs versus 17 FEs) than on trials with a left distractor (35 ICs versus 28 FEs), indicated by a significant association between distractor side and error type $\left(\chi^{2}(1)=25.40, p<.0001\right)$. The frequency of ICs did not differ as a function of whether they involved the target colour and distractor letter or the target letter and distractor colour $(p>.05)$.

\section{RT analysis for ICs and FEs}

To assess errors as a function of the time at which the patient responded, we rank ordered all RTs, including those of correct and incorrect responses, and then computed the $20^{\text {th }}, 40^{\text {th }}, 60^{\text {th }}$ and $80^{\text {th }}$ percentiles. This procedure allowed us to divide the data into five time bins, each containing an equal number of trials. ICs and FEs were then assigned to the time bin for the trial when they occurred. The frequency of ICs and FEs in different time bins of the RT distribution are depicted in Figure 2. When a distractor appeared in the left field, ICs were mainly associated with short response latencies (Figure 2a). Test of binomial proportions showed that the proportion of ICs relative to FEs was larger than expected by chance in the first time bin $(z=$ 3.54 , exact $p<.0007)$, but not in the other time bins $(p>.05)$. When the distractor appeared in the right field, we observed a slightly higher proportion of ICs at short response latencies, but more importantly, a sudden increase in ICs in the latest time bin. However, the numbers of ICs were overwhelming compared with FEs (and highly significant using binomial proportions) for 
all time bins (Figure 2b).

The data were submitted to a 2-by-2-by-5 loglinear analysis, containing respectively distractor side, error type (ICs and FEs) and time bin as factors. The best fitting model contained all main effects along with the interaction between time bin and distractor side, and also the interaction between error type and distractor side (Likelihood Ratio $\left(\right.$ L.R.) $\left.\chi^{2}(8)=8.24, p=.41\right)$. The first interaction reflected the observation that the number of errors FEs increased in later time bins in trials with a right distractor, while this was not the case in trials with a left distractor $\left(\chi^{2}(4)=18.04, p=.001\right)$. The interaction between error type and distractor side was due to the proportion of ICs being larger for trials with a right distractor $\left(\chi^{2}(1)=23.96, p<.0001\right)$. Although the absolute frequency of FEs did not differ across time bins (left distractor: $\chi^{2}(4)=$ 3.07 , exact $p=.59$; right distractor: $\chi^{2}(4)=4.47$, exact $\left.p=.38\right)$, the frequency of ICs did. With a left distractor ICs decreased in the longer time bin $\left(\chi^{2}(4)=8.86\right.$, exact $p=.07$ ), with a right distractor the proportions of ICs first decreased and then increased in the longer time bins $\left(\chi^{2}(4)\right.$ $=45.58, p<.0001)$.

(Insert Figure 2 about here)

\section{Supplementary analyses}

In the RT analysis presented above, time bins were calculated using RTs generated by all trials, in order to improve their reliability. However, we also calculated time bins separately for trials when distractors fell in GK's right and left visual field. Following this procedure, the best fitting model in the loglinear analysis $\left(L \cdot R \cdot \chi^{2}(8)=7.92, p=.44\right)$ also contained the interaction between distractor side and error type $\left(\chi^{2}(1)=23.96, p<.0001\right)$ along with the interaction between distractor side and time bin $\left(\chi^{2}(4)=12.15, p=.02\right)$. 
We also collected a limited dataset where GK made key presses himself. ICs were more likely to occur with a right distractor than with a left distractor $\left(\chi^{2}(1)=17.28, p<.0001\right)$. Moreover, 11 out of the 16 ICs made on trials with a right distractor were associated with the longest response latencies. This limited dataset adds to the validity of the datasets collected in the experiments.

\section{Distractor reports and other errors}

Distractor reports were more common when the distractor appeared in the right (236) compared to the left visual field $(17)\left(\chi^{2}(1)=189.57, p<.0001\right)$. 'Other' errors were also more likely to occur with a right distractor (17) than with a left distractor $(6)\left(\chi^{2}(1)=5.26, p=.02\right)$ (Figure 2).

\section{DISCUSSION}

The results reveal that, relative to FEs (reflecting just one feature from the target), GK made significant numbers of ICs in which he reported a feature from the distractor away from fixation along with a feature of the target at fixation. Under similar conditions, control participants make few errors ${ }^{2}$. We conclude that GK's performance is abnormal and is consistent with there being both poor spatial selection of the target at fixation, along with poor binding of the target's (and the distractor's) features in perception or in VSTM. This replicates prior data with GK (Humphreys et al., 2000) and with other patients with bilateral damage to posterior parietal cortex (Friedman-Hill et al., 1995).

In addition to showing reliable numbers of ICs, GK found it more difficult to identify a central target when it was flanked by a right distractor than when it was flanked by a left

\footnotetext{
${ }^{2}$ We tested this by running three age-matched controls (two female, aged 54 and 58; one male, aged 52) employing a more taxing procedure (presentation duration $175 \mathrm{~ms}$ and stimuli were followed by a $50 \mathrm{~ms}$ mask). The controls made five errors on 480 trials and one IC.
} 
distractor. This indicates that there was a spatial bias to selection, with the distractor item in the right field competing more strongly for selection than the distractor in the left field. This spatial bias in selection led sometimes to distractors being reported instead of the target, and this predominantly occurred when distractors fell in the right field. In addition, GK tended to make more ICs that reflected a feature from the right-field distractor than ICs reflecting a feature from a left-field distractor. This is consistent with the features from right-field items being more available for perceptual report (and creating competition with features from the target), compared with features from items in the left field.

Our analysis of when errors arose in the RT distribution revealed that there was a rise in ICs in the longest time bins when the distractor occurred in the right visual field. In addition, there was some evidence for ICs being relatively prevalent (compared with FEs) at the fastest time bin, but then decreasing over intermediate time intervals. Though this effect in the shortest time bin was reliable only with left-side distractors, there was a similar trend with right-side distractors and this was replicated in Experiment 2 below.

How can there be populations of 'fast' and 'slow' IC responses, with left-side features only occurring in the population of fast ICs? Several accounts can be put forward for the ICs in the population of slow responses. Here we consider three of them:

(1) Forgetting of feature bindings. GK may encode feature bindings correctly on many trials, but when his responses are slow, there may be time for these bindings to decay. This then leads to ICs occurring, with ICs increasing when there are long relative to short response latencies.

(2) Early right-side orienting. GK has a right-side bias in spatial selection, as indexed by the right-distractor errors evident in the data. Given this, we might expect that features are 
first extracted from right-side stimuli, and left-side features only become available for report later in time. When the distractor is in his right field, GK may first engage attention on the distractor and then try to re-orient attention to the left to report the target. When responses are slow, there may be sufficient time for GK to disengage from the right distractor to report some features of the target, but the features of the distractor may still be activated. As a consequence, ICs are generated. This can account for how ICs involving features from right-side distractors may increase when GK's responses are slow.

(3) Impaired consolidation. A third possibility is that GK is impaired in a slow-acting consolidation process that normally establishes a stable representation of any initial feature bindings that are formed (Cinel \& Humphreys, 2006). GK's brain lesion may mean that this consolidation process is noisy and disrupts the initial feature bindings that are formed. The consolidation process may also play a stronger role in report when the response latencies are slow, generating the increase in ICs in the slow response time bins. This impaired consolidation process may also be exacerbated by the right-side bias in spatial selection, if right-side features are strongly weighted when consolidation takes place. These right-side features are then imposed onto the initial feature representations, generating many ICs from right-side distractors.

In Experiments 2 and 3 we attempted to test these different alternative accounts. Experiment 2 used a forced-choice response procedure, which ought to minimise ICs due to forgetting (account (1) above; see Treisman \& Schmidt, 1982). On the other hand, effects due to early orienting to a right-side distractor, or effects due to later-acting consolidation processes, should still be apparent. In Experiment 3 we varied the actual exposure durations for stimuli. If there is initial orienting to right-side stimuli, followed by left-side encoding of features, then ICs should decrease when longer exposures allow left-side features to be encoded and re-orienting of 
Decomposing visual binding over time

attention to take place.

Whichever account is put forward to explain the ICs at long response latencies, it is difficult to see how it might also account for the ICs in the population of fast responses. These ICs suggest that GK does initially extract left-side features (from the target, with a right-side distractor, or from a left-side distractor when it is present), and there is not a complete capture of attention by a right-side item. These left-side features appear to be available early enough to influence GK's fastest responses. Given their occurrence in the fastest time bins, these ICs are unlikely to reflect initial right-side orienting, memory failures, or a deficit in re-entrant (attentional) consolidation. Instead, we propose that these ICs reflect the incorrect initial binding of perceptual features. We suggest that GK extracts features from both left- and right-side items, but, on some proportion of trials, these features are mis-combined at an initial stage of encoding a bound representation. The possibility of initial binding errors was tested further under forcedchoice procedures in Experiment 2 and by varying the exposure duration in Experiment 3. The proportion of ICs reflecting initial feature mis-combinations may be relatively large with short exposures, when later acting processes (attentional re-orienting, forgetting from memory or impaired consolidation) may not contribute to performance.

\section{EXPERIMENT 2: Reducing memory failures through a two-alternative forced-choice task}

Experiment 2 provided a test whether ICs arose purely due to the loss of information in memory about which features had initially been coded. To do this, we reduced the load of the response task by asking GK to make a two alternative forced-choice response to stimuli (Is there a [particular coloured letter] present at fixation?). Prior work indicates that the forced-choice task reduces the load of memorising both features of a target, since participants only have to verify 
the presence of features instead of having to maintain them while making the response (see Humphreys et al., 2000). If the length of time that items are held in memory is critical, then we expect the relative proportion of ICs to reduce here.

\section{METHOD}

Only two-letter trials were used, either with a distractor in the right visual field or with a left distractor. The letters and colours were selected from a set of three values, respectively A/B/C and blue/green/red. Each trial started with a fixation cross in the centre of the screen (1000 ms), followed by the presentation of a probe $(1800 \mathrm{~ms})$, a delay $(500 \mathrm{~ms})$, the stimulus display $(250$ ms) and a response window. Instead of having to report the letter and the colour of the target at fixation, GK was asked if a pre-specified letter-colour combination was present or absent in the target at fixation. To minimise misidentification of the probe, the experimenter read aloud the colour and the letter of the probe. GK gave his answer orally ('same' or 'different') and the experimenter registered RTs by key presses. On same identity probe trials, the probe contained the letter-colour combination which was present in the target. On IC probe trials we presented a coloured letter constructed by recombining the colour or the letter of the target with respectively the letter or the colour of the distractor. On FE probe trials we presented a coloured letter, constructed by recombining the colour or the letter of target with respectively the letter or the colour not present in the display. If ICs are perceptual in nature, there should be a larger number of false positive responses to IC than to $F E$ probes. If ICs are due to memory failures, then differences between the conditions should be reduced, if not eliminated. Each block contained 72 trials, 36 with a distractor on the left and 36 with a distractor on the right of the target. We presented an equal number of same identity, IC and FE probes in each block $(n=24)$. Hence, a 
block contained fewer target present (33\%) than target absent probes (67\%). We ran 12 blocks, leading to 144 trials in each condition.

\section{RESULTS}

\section{Overall performance}

GK gave the correct answer on $78 \%$ of the trials. Accuracy was equally good for trials with a left and for trials with a right distractor $(p>.05)$. GK was better at rejecting a FE probe $(88 \%$ correct) and accepting an same identity probe ( $82 \%$ correct) than he was at rejecting an IC probe $(64 \%$ correct $)\left(\chi^{2}(2)=51.36, p<.0001\right)$.

\section{ICs versus FEs}

When presented with a same identity probe, GK was significantly above chance in accepting it ( $\mathrm{z}$ $=10.96, p<.0001)$, regardless of whether the trial contained a right or a left distractor $\left(\chi^{2}(1)=\right.$ $.21, p=.64)$. The frequency of `same` and 'different` responses on FE and IC probe trials were submitted to a loglinear analysis with distractor side (left - right), probe type (FE - IC) and response (same - different) as the defining variables. The best model contained the interaction between probe type and response $\left(L . R . \chi^{2}(4)=.41, p=.98\right)$. As illustrated in Figure 3, GK was more likely to reject a FE probe than he was to reject an IC probe $\left(\chi^{2}(1)=45.62, \mathrm{p}<.0001\right)$. We did not find any evidence for a main effect of distractor side, neither for an interaction between probe type and distractor side $(p>.05)$.

\section{(Insert Figure 3 about here)}

\section{RT analysis of ICs and FEs}

We divided the range of response latencies into five equal time bins. ICs were defined as `same` 
responses to conjunction probes and FEs as `same` responses to feature probes. The data were pooled across distractor side as this factor did not affect performance here. As can be seen in Figure 4, FEs were equally distributed across all portions of the RT distribution $\left(\chi^{2}(4)=3.71\right.$, exact $p=.48)$. ICs, however, tended to be associated with short response latencies $\left(\chi^{2}(4)=7.92\right.$, $p=.09$ ). The proportion of ICs was larger in the first time bin, compared to each other time bin

(second: $\chi^{2}(1)=4.97, p=.03$; third: $\chi^{2}(1)=7.45, p=.0063$; fourth: $\chi^{2}(1)=3.96, p<.05$; fifth: $\left.\chi^{2}(1)=3.96, p<.05\right)$

(Insert Figure 4 about here)

\section{DISCUSSION}

There are several critical points to note. One is that the forced-choice procedure eliminated the differences in performance previously observed with left and right distractors. If there was automatic capture of attention by a right-side stimulus, then target detection should be worse with a right- than a left-distractor irrespective of whether or not a forced-choice identification response was required. The data do not fit with that but suggest instead that the top-down set to the target's features reduced the spatially-selective bias in GK's performance. Single cell recording studies in the monkey have shown that feature priming can influence the competition for selection between visual features. Chelazzi, Miller, Duncan and Desimone (1993) reported that neurons in the inferior temporal cortex were activated more rapidly by features consistent with a prime held in memory than by features inconsistent with the prior stimulus. Soto and Humphreys (2006) further demonstrated that visual extinction in patients could be reduced when patients held an item in memory and this matched the item in the otherwise extinguished field. In our case, the probe would need to be held in working memory to 
Decomposing visual binding over time

verify against the target. As in Soto and Humphreys (2006), the apparent top-down priming from working memory counter-acted the spatial bias in selection. Our data here suggest that the spatial bias emerges when GK attempts to identify (as in Experiment 1) rather than verify (as here) target features, perhaps because the right-field bias emerges over time when identification is prolonged.

The second result to note is that GK appeared biased to respond 'same', leading to higher performance on same identity trials than expected from the errors on mis-match trials (particularly with IC probes). More importantly though, GK made false positives to IC probes and these were more frequent than false positives to FE probes. That is, there was evidence for ICs occurring, even though a forced-choice procedure was adopted. The results indicate that at least some ICs are perceptual in nature, a conclusion that has already been reported for normal subjects by Treisman and Schmidt (1982) and for GK by Humphreys et al. (2000). ICs made in this forced-choice detection task are unlikely to reflect post-perceptual decision strategies or decay/loss of consolidated representations in VSTM. There was a minimal VSTM load and the task only required that a briefly presented letter at fixation had to be verified against the probe. Under these conditions, it is unlikely that ICs reflect conjunctive information being forgotten over time.

The third noteworthy finding is that the ICs that did occur were most frequent when GK made (for him) fast responses to stimuli. This fits with the argument that the ICs emerging at the slow-end of the response distribution, in Experiment 1, reflect a late influence effect of rightfield distractors which is minimised under forced-choice conditions. One account of this is that the right-field distractors affect a late consolidation process, which operates in a top-down manner to stabilise initial feature bindings. The forced-choice verification procedure may reduce 
the need for consolidation, since the primed representation of the target in working memory may serve the purpose of stabilising the initial representation of the stimulus. The ICs at the fast end remained here, though, even though any consolidation process seemed to be less critical. This is consistent with these 'fast ICs reflecting poor initial binding prior to verification from working memory taking place.

In Experiment 3 we used a different procedure to examine the time course of feature binding. In this experiment we directly manipulated the time GK had available to process the stimuli. We propose that perceptual mis-bindings should occur even under short presentation conditions since they reflect error occurring at an early stage of visual encoding. However slow consolidation processes may contribute more strongly to performance when exposures are prolonged. Impairments at this consolidation stage, then, may emerge when durations are more prolonged.

\section{EXPERIMENT 3: The occurrence of ICs across various exposure durations}

\section{METHOD}

Unless otherwise noted, the Method was the same as for the previous experiments. GK was run in four blocks of trials in an ABBA design. On each trial he received a fixation cross (2000 ms), followed by a probe (2000 ms) and, depending on the block, an exposure of the stimulus display for either 200ms (short exposure duration) or $2000 \mathrm{~ms}$ (long exposure duration). As in Experiment 2, the probe was read aloud by the experimenter, to ensure that GK encoded the features he had to verify. Each display contained one of five letters (E/H/K/T/R) in one of five colours (black/blue/green/red,/yellow) at fixation, and a second stimulus, with a different letter/colour combination in his right visual field. The size of the letters and the distances apart 
matched those used in Experiment 1. The probe specified a possible target at fixation. There were same identity probes (e.g., red T? on a trial where a red $\mathrm{T}$ fell at fixation), $I C$ probes which combined the colour/shape of the item at fixation with the shape/colour of the distractor (e.g., a probe green $\mathrm{T}$ ? when a red $\mathrm{T}$ fell at fixation and a green $\mathrm{R}$ in GK's right field), and $F E$ probes (e.g., a probe red $\mathrm{H}$ ? when a red $\mathrm{T}$ fell at fixation and a green $\mathrm{R}$ distractor was present). In one duration block there were 100 same identity probes, 100 IC probes and 100 FE probes, creating 1200 trials altogether across the blocks. The FE probes were determined by randomly sampling the set of possible items with the constraint that one of the features of the probe matched a feature of the target and the other did not match any other feature in the display. Only right-field distractors were used here to maximise the number of errors. Responses were not timed, but after every trial GK was asked to report whether he was certain or uncertain/guessing.

\section{RESULTS}

GK made $66 \%$ correct responses on same identity trials with a 200 ms exposure duration and $83 \%$ correct responses with a $2000 \mathrm{~ms}$ exposure. Correct reports improved across time $\left(\chi^{2}(1)=18.54, \mathrm{p}<.001\right)$. However, the certainty of these correct verifications did not vary. $74 / 128$ $(58 \%)$ of GK's correct responses at the short duration were certain, and 104/166 (63\%) of the correct responses were certain at the longer duration $(p>.05)$.

The number of IC and FE errors (false positive responses to IC and FE probes), along with the certainty of those responses, are given in Figure 5a and 5b. At both the short and the

long exposure durations, there were more IC than FE errors (short exposure: $\chi^{2}(1)=11.81, p<$ .001 ; long exposure: $\left.\chi^{2}(1)=39.02, p<.001\right)$. GK made more ICs at long relative to short stimulus durations $\left(\chi^{2}(1)=13.62, p<.001\right)$, while the number of FE tended to decrease over 
Decomposing visual binding over time

time $\left(\chi^{2}(1)=3.47, p=.06\right)$.

For IC errors there was a significant change in the pattern of certain to uncertain errors across the two exposure durations $\left(\chi^{2}(1)=40.97, p<.001\right)$. The number of responses rated as certain decreased as the exposure duration increased $\left(\chi^{2}(1)=11.64, p<.001\right)$, while the number of uncertain responses increased $\left(\chi^{2}(1)=12.04, p<.001\right)$. In contrast to the results for IC errors, the pattern of certain to uncertain errors did not change for FEs across the two exposure durations $(p>.05)$.

At the short exposure there were more certain ICs than certain FEs $\left(\chi^{2}(1)=6.95, \mathrm{p}<\right.$ $.01)$, while, for uncertain responses, the two types of error did not differ $(p>.05)$. At the long exposure there were more uncertain ICs than FEs $\left(\chi^{2}(1)=15.9, p<.01\right)$ while there were no differences in the number of certain ICs and FEs $(p>.05)$.

(insert Figure 5 about here)

\section{DISCUSSION}

Across both exposure durations there were more ICs than FEs, indicating a deficit in binding rather than just feature encoding. More interestingly, the pattern of ICs and FEs also changed as a function of the exposure duration. FEs generally decreased as the exposure duration increased, and correct verification responses to targets became more likely, consistent with features being better encoded and any initial binding operation being more successful. In contrast, ICs increased over time. In addition, the proportion of 'certain' to 'uncertain' ICs changed - there were more certain than uncertain ICs with the short exposure, while at the longer exposure there were more uncertain than certain ICs.

Let us consider how these data fit with the accounts of ICs we have reviewed. As with 
Experiment 2, the present results are difficult to reconcile with an account in terms of bindings being formed but then being lost from VSTM. In this study we again used a forced-choice procedure with minimal load on VSTM. This should minimise ICs due to loss of information in VSTM, yet we again found clear evidence for ICs occurring.

A second account proposed that there might be initial orienting of attention to a right-side distractor, with ICs occurring when GK disengages attention from the distractor to attend to the target but there is a confusion of target features with those activated by early orienting to the distractor. This account could predict that ICs could reliably occur at both exposure durations used here, depending on when attention disengages from the distractor to the target. Also ICs could increase at the longer duration if this allows more time for the disengagement process to occur. However, this account has no explanation for why 'certain' ICs tended to arise with the short exposure while 'uncertain' ICs increased at the longer duration.

The third account is that there are two sources of mis-binding in patient GK. The first reflects an error at an initial feature binding stage, which occurs rapidly. The second reflects an error in a slower-acting consolidation process, which is noisy and may also be influenced by a selection bias favouring right-side distractors. This two-process account attributes the errors with short exposures to mis-binding at the initial stage. We suggest that GK is able to make perceptual reports at short exposures when the features are well encoded, and that he is relatively certain he is correct on such occasions - even when the features are mis-combined to create ICs. With longer exposures, the consolidation processes comes into operation, normally to stabilise the initial representation of the stimulus. However, if the processs is noisy, and if it is strongly influenced by the activated features of right-side distractors, then it can create ICs. Due to the process being noisy, GK is uncertain when these errors occur. 
We can also consider these data in relation to other accounts that assume there is a single binding process based on GK focusing attention on the target. Currently the most influential account of visual binding is probably that offered by Feature Integration Theory (FIT). According to FIT, feature binding is dependent on the application of attention to the locations where features fall, with attention affecting the initial coding of visual elements and the filtering out of irrelevant distractors (Treisman, 1998). Attention is held to be modulated through the posterior parietal cortex, and is impaired in patients such as GK. Disrupting attention can have two effects - it can impair coding of the basic features of stimuli, and it can disrupt feature binding because the features of distractors remain available to be combined with those of the target. In contrast to Treisman's FIT, we suggest that ICs can be elicited either by mis-binding in the first place ('early' ICs which produce a percept as strong as real binding, see also the results about certainty in Experiment 3) or by disintegration of features over time, which is a result of a deficit in the consolidation process. Only this last account resembles that of FIT. According to FIT, attention is a necessary condition for conjunctive feature coding to occur. However, we propose that initial perceptual binding can be based on conjunctive coding units, which are then reinforced by attention. Hence, while FIT predicts that ICs only occur when attention is overloaded (e.g. by briefly presenting multiple masked items in healthy volunteers (Treisman \& Schmidt, 1982), or by brain damage in patients with Bàlint's syndrome), we suggest that ICs may also be reported when the initial binding is noisy.

One other variant of a single process account can also be raised. This is that GK employed a response bias which changed across the exposure durations. The argument here is that GK employs a bias to say 'yes' when both features are present, given that accuracy on target present trials was higher than expected from a combination of FEs and ICs. To explain the 
increased accuracy on target present trials across time, we can argue that GK guesses 'no' when he is uncertain and the duration is $200 \mathrm{~ms}$, and he guesses 'yes' when he is uncertain and the duration is $2000 \mathrm{~ms}$. The increase in uncertain ICs, across time, might be attributed to this shift in response bias. However, according to this account we should expect that GK would make more FEs at the longer duration, due to incorrect 'yes' guesses. This is the opposite to the pattern of data we observed.

Overall we conclude that the pattern of results from Experiment 3 matches better the two process account which holds that there are two factors contributing to GK's ICs: poor initial binding and impaired consolidation of bindings. The initial binding process is highlighted at the short exposure, and, when the initial binding process is incorrect, it leads to ICs that GK is confident about. At the longer exposure, the contribution from consolidation processes increases. GK's impairment in consolidation generates ICs at the longer duration, but these errors are made with low certainty. According to this account, the increase in 'uncertain ICs' across time reflects the contribution from the impaired consolidation process.

The present data with GK replicate the results reported by Braet and Humphreys (2009) with another simultanagnosic patient, PF. Like GK, PF made abnormal numbers of ICs at both long and short exposures, but the number of ICs again increased at the longer durations. Braet and Humphreys also found that TMS to posterior parietal cortex induced ICs in normal participants at exposures later than those that generated FEs, suggesting disruption to a slow binding process that followed feature coding. This fits with the idea that disruption to neural activity in posterior parietal cortex affects the slow consolidation of bindings between visual features. 


\section{GENERAL DISCUSSION}

We have presented evidence that GK, a patient with posterior parietal lesions, makes IC errors at a greater rate than FEs, using presentation durations where few errors are made by normal participants. Moreover, these ICs occur under forced-choice (Experiments 2 and 3) as well as free report conditions (Experiment 1), suggesting that they are perceptual in origin and not due to the loss of bound representation in VSTM. On top of this, we also demonstrated that: (1) ICs were elicited at different times when GK responded, with a relatively high prevalence of ICs occurring within his fastest responses followed by an increased number of ICs during GK's slowest responses (Experiment 1); (2) in Experiment 1 ICs were most prevalent when a distractor fell in GK's right visual field, consistent with GK having a rightwards spatial bias in selection (see Gilchrist et al., 1996); these right-field distractors contributed to GK's ICs particularly when he responded slowly; (3) the stronger effect of right-side distractors was eliminated under forcedchoice conditions (Experiment 2), as were the increased numbers of ICs at slow end of GK's response distribution; and (4) there was a relatively high frequency of ICs responses that GK was certain of when there were relatively short stimulus exposures, while uncertain ICs actually increased when the exposure duration increased (Experiment 3).

We have considered several accounts of the observed pattern of ICs over time. One possibility is that features are bound normally at a perceptual level, but these feature bindings are then lost from VSTM. This account has difficulty explaining the ICs that occurred under forcedchoice conditions, and that ICs should increase as the exposure duration lengthens (given that, at longer exposures, items should be better encoded into memory; Experiment 3). A second possibility is that GK automatically attends to a right-side stimulus and then disengages attention over time. When there is a right-field distractor, there is initial activation of the distractor's 
features which then compete with those made available when attention engages on the target. ICs result and they are associated with slow responses emerging only after the re-engagement of attention on the target. This possibility fits with some of the results from Experiment 1, but then has difficulty explaining other aspects of the data. One is that there were ICs at the fast end of GK's response distribution, from left as well as right-side distractors. These 'fast' ICs from leftside distractors do not fit with attentional capture by right-side distractors. In addition, automatic capture and attentional disengagement should generate ICs even under forced-choice conditions. However under forced-choice conditions there were no more ICs from right- than from left-side distractors, and there was no longer any increase in ICs at the slow end of GK's response latencies (Experiment 2). A third problem is that it is not clear why, with short exposures, GK should make ICs where he has high perceptual certainty, which then decrease as the exposure duration lengthens (Experiment 3). Again this account does not appear to stand up. We also reviewed how well a single process account of feature binding, such as FIT, could accommodate the data. The data from Experiment 3 are problematic for this account. In Experiment 3 FEs decreased as the exposure duration increased. This is consistent with GK being better able to fix his attention on the target at longer exposures. This should also lead to a reduction in ICs. In contrast to this, (uncertain) ICs increased as stimulus exposures lengthened.

A multi-stage account of binding.

The different patterns of data apparent here can be accommodated by the multi-stage account of binding (Cinel \& Humphreys, 2006). This account states that there is an initial stage of feature binding which supports the reporting of feature conjunctions but which is not very stable. This provides a 'quick and dirty' representation of the visual world. Over time these 
bindings are (normally) consolidated by a top-down feedback process, which seeks to confirm the initial representations by a re-entrant feedback process. This may be realised computationally through a process such as 'adaptive resonance' (Grossberg, 1987; see also Hochstein \& Ahissar, 2002), where a coarse initial representation is read-out of and then fed-back to early perceptual representations. A framework for this idea is presented in Figure 6. In adaptive resonance models, the feedback process helps to consolidate a stable representation of the input. We will term this 'perceptual consolidation', to distinguish the idea from consolidation processes based on a template for an expected target, discussed below. We propose that perceptual consolidation is not necessary for perceptual report, but rather it forms more robust bindings that generate better perceptual report. However, perceptual report can be based on information made available from early visual representations without consolidation taking place. We propose that GK has impairments in both the initial feature binding process and in the slower, re-entrant process of perceptual consolidation. The impaired initial binding process leads to incorrect conjunctions being formed from the outset. This process operates rapidly and survives short exposures. When GK responded rapidly, the information available will be weighted towards these initial bindings. Experiment 3 also indicates that GK had high perceptual certainty when ICs were made to stimuli with short exposures. We propose that his perceptual certainty is increased when the later, re-entrant consolidation process has not had time to affect performance since, in GK's case, we believe that this process too is impaired and adds noise to his perceptual representations. The addition of noise to the initial representations means that features belonging to different stimuli co-activate common units, increasing the likelihood of ICs. Since perceptual consolidation operates after any initial feature binding, then ICs from the consolidation process will be strongly weighted in GK's slower responses (Experiment 1) and these ICs will be apparent when the 
Decomposing visual binding over time

exposure durations are sufficiently long to enable perceptual consolidation to take place (Experiment 3). GK may be uncertain about ICs from impaired perceptual consolidation, since his initial representations will be made noisier when the process takes place. It is important to stress here that patients with Bàlint's syndrome experience prevalent binding deficits in everyday life, even when fully attending objects without time constraints.

\section{(insert Figure 6 about here)}

The data from Experiment 1 also suggest that, for GK, the 'slow' ICs often reflected features from right-side distractors. This raises the interesting possibility that the perceptual consolidation process was more spatially biased than the initial binding process, where differences between left- and right-side distractors were less apparent. We suggest that, when reentrant feedback took place, features from right-side distractors were strongly weighted, and tended to be used in matching against the perceptual input. ICs would result. This is represented by the graded shading for the higher-level re-entrant processes in Figure 6.

One other interesting result to note is that both the increased ICs associated with slow RTs, and the bias for right- over left-side distractors, were eliminated when a forced-choice verification task was employed (Experiment 2). To account for this we propose that, in the forced-choice condition, GK could use a template of the target coded in working memory to consolidate bindings formed at the first stage of conjunctive coding. This 'top-down consolidation' process reduced the influence of perceptual consolidation on performance, eliminating many slow ICs and the right-side bias in perceptual consolidation. Previous studies have demonstrated that top-down consolidation can operate in patients with posterior parietal damage, and that it can help to overcome spatial biases in visual selection (Soto \& Humphreys, 
2006).

$\underline{\text { Time course and response times. }}$

In Experiments 1 and 2 we carried out an initial analysis of the time course of feature binding by analysing the times at which ICs were generated, when GK responded. This analysis is based on the assumption that the information supporting 'late' responses has been available for longer than the information supporting responses on which 'early' responses are based. Our argument here is that there is a monotonic relationship between the length of time over which processes such as stimulus encoding and consolidation take place and the time at which GK responded; however we do not assume that his reaction times exactly reflect when encoding and consolidation occur. Nevertheless we note that previous work with GK (and indeed other patients with brain lesions) has shown that information processing can be slowed (see Duncan et al., 2003), which makes it difficult to gauge exactly when binding processes would operate, since these processes may be slowed along with his general reaction times. However, the point of Experiment 3 was to provide converging evidence where we specifically manipulated the time for which stimuli are available. The results match those generated by our analysis of errors as a function of the time of response, giving support to our account of the error-RT analyses in Experiments 1 and 2.

\section{$\underline{\text { Lesion sites, visual localisation and attention }}$}

Binding problems associated with posterior parietal damage may occur for a variety of reasons. One possibility is that there is poor localisation of stimuli, since neurons in posterior parietal cortex may contain a spatial map of the visual world (cf. Treisman, 1998). Damage to 
units coding spatial location lead to poor co-alignment of shape and colour features within the units that code conjunctive relations and hence conjunction errors occur. This argument is consistent with the coarse coding account of McCrae, Buxbaum and Coslett (2006), who proposed that the ICs made by their patient with bilateral posterior lesions reflected poor coding of visual feature locations. Poor location coding, during both the initial registering of features and in the later consolidation of feature relations, could explain the two patterns of ICs we have observed. For example, GK had lesions affecting both posterior occipito-parietal cortex more anterior parietal cortex. The posterior lesions may lead to poor initial registration of feature locations (and 'fast ICs'), while poor alignment across features, and subsequent impairment to re-entrant feedback processes, comes about from loss of location information in more anterior parietal regions, so that activation spreads across units that should be activated by different objects; 'slow ICs' then result.

Humphreys (2001) (also Humphreys et al., 2009) distinguished between binding within the shape dimension (in the ventral visual stream) and binding shape and surface properties (reliant on interactions between the ventral and dorsal visual streams). Here we provide evidence for the proposal that distinct binding deficits may occur even within the process of binding shape and surface properties - distinguishing fast, initial binding from slower re-entrant perceptual consolidation. We may also think of this proposed re-entrant process in terms of attention being paid to the locations where the to-be-bound features occur, though whether the consolidation of feature-bindings is limited to only one spatial region at a time (e.g., as proposed by FIT) is a moot point (see Kubovy \& Cohen, 2001; Kubovy, Cohen, \& Hollier, 1999).

On our account, patients with posterior parietal damage can have difficulty in forming stable perceptual representations to enter into VSTM. Coslett and Saffran (1991), on the other 
hand, have argued that such patients have an impaired representation of information within VSTM. They argued that impairments within VSTM itself could explain why such patients are sometimes better at reporting two related objects than two unrelated objects, proposing that semantic relations between stimuli can help sustain a representation in VSTM. We propose that the problem is not following encoding in VSTM, though, but in stabilising initial encoding into VSTM through top-down feedback from posterior parietal cortex. Possibly, the binding of information is helped when items are semantically related because a top-down consolidation

process can then play a stronger role than the operation of perceptual consolidation outlined in Figure 6, which we suggest is impaired after posterior parietal damage.

\section{CONCLUSION}

We have presented evidence for ICs arising at least two stages in visual processing, due to (1) errors in initial perceptual binding and (2) errors in subsequent perceptual consolidation of binding. These results add evidence to the hypothesis that visual binding involves multiple operations and is not a single-step process (Cinel \& Humphreys, 2006). 
Decomposing visual binding over time

\section{Reference List}

Braet, W., \& Humphreys, G. W. (2009). The role of re-entrant processes in feature binding: Evidence from neuropsychology and TMS on late onset illusory conjunctions. Visual Cognition, 17, 25-47.

Chelazzi, L., Miller, E. K., Duncan, J., \& Desimone, R. (1993). A neural basis for visual search in inferior temporal cortex. Nature, 363, 345-347.

Chun, M.M., \& Potter, M.C. (1995). A two-stage model for multiple target detection in rapid serial visual presentation. Journal of Experimental Psychology: Human Perception and Performance, 21,109-127.

Cinel, C., \& Humphreys, G. W. (2006). On the relations between implicit and explicit spatial binding: Evidence from Balint`s syndrome. Cognitive, Affective and Behavioral Neuroscience, 6, 127-140.

Cohen, A., \& Ivry, R. (1989). Illusory conjunctions inside and outside the focus of attention. Journal of Experimental Psychology-Human Perception and Performance, 15, 650-663.

Cohen, A., \& Rafal, R. D. (1991). Attention and feature integration - Illusory conjunctions in a patient with a parietal lobe lesion. Psychological Science, 2, 106-110.

Coslett, H. B., \& Saffran, E. (1991). Simultanagnosia - To see but not 2 see. Brain, 114, 1523-1545.

Donk, M. (2001). Illusory conjunctions die hard: A reply to Prinzmetal, Diedrichsen, and Ivry (2001). Journal of Experimental Psychology-Human Perception and Performance, 27, 542-546.

Duncan, J., \& Humphreys, G. W. (1989). Visual-search and stimulus similarity. Psychological Review, 96, 433-458.

Duncan, J., Bundesen, C., Olson, A., Humphreys, G., Ward, R., Kyllingsbaek, S., Van Raamsdonk, M., Rorden, C. \& Chavda, S. (2003).Attentional functions in dorsal and ventral simultanagnosia. Cognitive Neuropsychology, 20, 675-701

Friedman-Hill, S. R., Robertson, L. C., \& Treisman, A. (1995). Parietal contributions to visual feature 
Decomposing visual binding over time

binding - Evidence from a patient with bilateral lesions. Science, 269, 853-855.

Gilchrist, I. D., Humphreys, G. W., \& Riddoch, M. J. (1996). Grouping and extinction: Evidence for lowlevel modulation of visual selection. Cognitive Neuropsychology, 13, 1223-1249.

Grossberg, S. (1987) Competitive learning: From interactive activation to adaptive resonance. Cognitive Science, 11, 23-63.

Hochstein, S., \& Ahissar, M. (2002). View from the top: Hierarchies and reverse hierarchies in the visual system. Neuron, 36, 791-804.

Hubel, D. H., \& Livingstone, M. S. (1987). Segregation of form, color, and stereopsis in primate area 18. Journal of Neuroscience, 7, 3378-3415.

Humphreys, G. W. (1999). Case Studies in the Neuropsychology of Vision. Hove: Psychology Press.

Humphreys, G. W. (1998). Neural representation of objects in space: A dual coding account. Philosophical Transactions of the Royal Society of London Series B-Biological Sciences, 353, 13411351.

Humphreys, G. W. (2001). A multi-stage account of binding in vision: Neuropsychological evidence. Visual Cognition, 8, 381-410.

Humphreys, G. W., Cinel, C., Wolfe, J., Olson, A., \& Klempen, N. (2000). Fractionating the binding process: Neuropsychological evidence distinguishing binding of form from binding of surface features. Vision Research, 40, 1569-1596.

Humphreys, G.W., Hodsoll, J. \& Riddoch, M.J. (2009). Fractionating the binding process: Neuropsychological evidence from reversed search efficiencies. Journal of Experimental Psychology: Human Perception and Performance, 35, 627-647.

Humphreys, G. W., \& Riddoch, M. J. (2003). From what to where: Neuropsychological evidence for 
Decomposing visual binding over time

implicit interactions between object- and space-based attention. Psychological Science, 14, 487-492.

Kubovy, M., \& Cohen, D. J. (2001). What boundaries tell us about binding. Trends in Cognitive Sciences, 5, 93-95.

Kubovy, M., Cohen, D. J., \& Hollier, J. (1999). Feature integration that routinely occurs without focal attention. Psychonomic Bulletin \& Review, 6, 183-203.

Kumada, T., \& Humphreys, G. W. (2001). Lexical recovery from extinction: Interactions between visual form and stored knowledge modulate visual selection. Cognitive Neuropsychology, 18, 465-478.

Luck, S. J., \& Vogel, E. K. (1997). The capacity of visual working memory for features and conjunctions. Nature, 390, 279-281.

Mccrea, S. M., Buxbaum, L. J., \& Coslett, H. B. (2006). Illusory conjunctions in simultanagnosia: Coarse coding of visual feature location? Neuropsychologia, 44, 1724-1736.

Robertson, L., Treisman, A., Friedman-Hill, S. R., \& Grabowecky, M. (1997). The interaction of spatial and object pathways: Evidence from Balint's syndrome. Journal of Cognitive Neuroscience, 9, 295-317.

Robertson, L. C. (2003). Binding, spatial attention and perceptual awareness. Nature Reviews Neuroscience, 4, 93-102.

Soto, D., \& Humphreys, G. W. (2006). Seeing the content of the mind: Enhanced awareness through working memory in patients with visual extinction. Proceedings of the National Academy of Sciences of the United States of America, 103, 4789-4792.

Treisman, A. (1998). Feature binding, attention and object perception. Philosophical Transactions of the Royal Society of London Series B-Biological Sciences, 353, 1295-1306.

Treisman, A., \& Schmidt, H. (1982). Illusory conjunctions in the perception of objects. Cognitive Psychology, 14, 107-141. 
Decomposing visual binding over time

Ward, R., Goodrich, S. J., \& Driver, J. (1994). Grouping reduces visual extinction: Neuropsychological evidence for weight-linkage in visual selection. Visual Cognition, 1, 101-129.

Wheeler, M. E., \& Treisman, A. M. (2002). Binding in short-term visual memory. Journal of Experimental Psychology-General, 131, 48-64.

Wojciulik, E., \& Kanwisher, N. (1998). Implicit but not explicit feature binding in a Balint's patient. Visual Cognition, 5, 157-181. 


\section{FIGURE CAPTIONS}

Figure 1. Experiment 1: (a) Example of a two-letter trial with a target at fixation (e.g., red A) and a distractor in the right visual field (e.g., green B). The different shading patterns in the letters reflect different colours. (b) Illustration of the different answers that GK may give in response to the stimulus display presented in (a). Different types of errors can be made: illusory conjunctions (ICs, e.g. red B), feature errors (FEs, e.g., red C), distractor reports (green B), and 'other' errors (e.g., blue C).

Figure 2. $\quad$ Experiment 1: The distribution of the correct responses and the four different error types (ICs, FEs, distractor reports, other errors) in the five time bins, $(a)$ for trials with a left field distractor, and (b) for trials with a right field distractor.

Figure 3. $\quad$ Experiment 2: The frequency of 'same' and 'different' answers in response to same identity, FE and IC probes, (a) for trials with a distractor on the left, and $(b)$ for trials with a distractor on the right. The dashed line reflects chance. Correct answers are 'same' responses to same identity probes, and 'different' responses to FE and IC probes. Incorrect answers are 'different' responses to same identity probes (false negatives) and 'same' responses to FE and IC probes (false positives).

Figure 4. $\quad$ Experiment 2: The distribution of correct responses and the three different error types (ICs: false positives to IC probes, FEs: false positives to FE probes, false negatives to same identity probes) in the five time bins.

Figure 5. $\quad$ Experiment 3: The frequency of false positive answers in response to IC probes $(c)$ and FE probes $(d)$, coloured according to GK's certainty about his answer.

Figure 6. A re-entrant framework for conjunctive coding. Separate input modules code colours and shapes at two different locations (1 and 2), and feed activation forward to conjunctive coding units that form initial feature bindings. These initial feature bindings are stabilised by re-entrant feedback of the initially activated representation. We assume that GK has impairments at both the first (feed-forward) stage of binding and at the later (feedback) stage. We also propose that the feedback stage was spatially biased to 'weight' activation from items in the right visual field, illustrated here by the grey-level gradient 
Decomposing visual binding over time

and the dotted feedback line from left-side units. Though we propose here local units for conjunctive coding, we note that conjunctive coding could take place through distributed representation over coarsely coded units. 
Decomposing visual binding over time

(a) Stimulus display

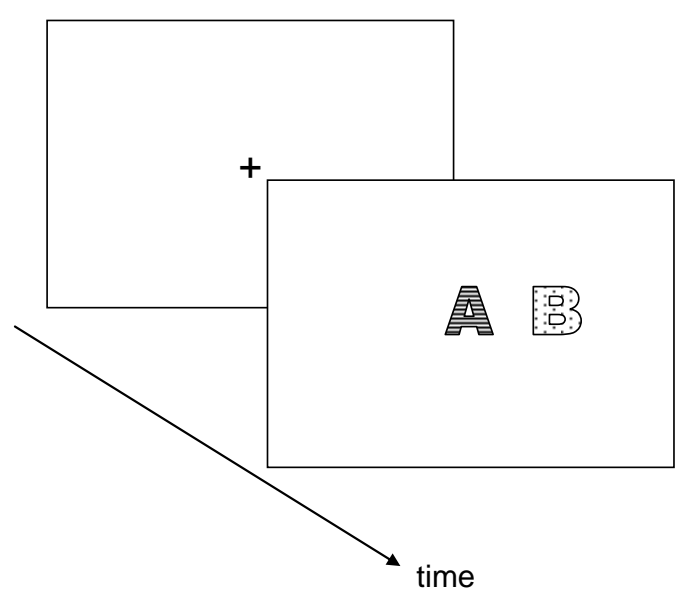

(b) Response options

CORRECT

correct shape \& colour 覓

INCORRECT

1. IC 临

2. $\mathrm{FE}$ 要 $\mathrm{A}$

3. distractor report

4. 'other' error, e.g. 8 D

Figure 1. Experiment 1: (a) Example of a two-letter trial with a target at fixation (e.g., red A) and a distractor in the right visual field (e.g., green B). The different shading patterns in the letters reflect different colours. (b) Illustration of the different answers that GK may give in response to the stimulus display presented in (a). Different types of errors can be made: illusory conjunctions (ICs, e.g. red B), feature errors (FEs, e.g., red C), distractor reports (green B), and 'other' errors (e.g., blue C). 


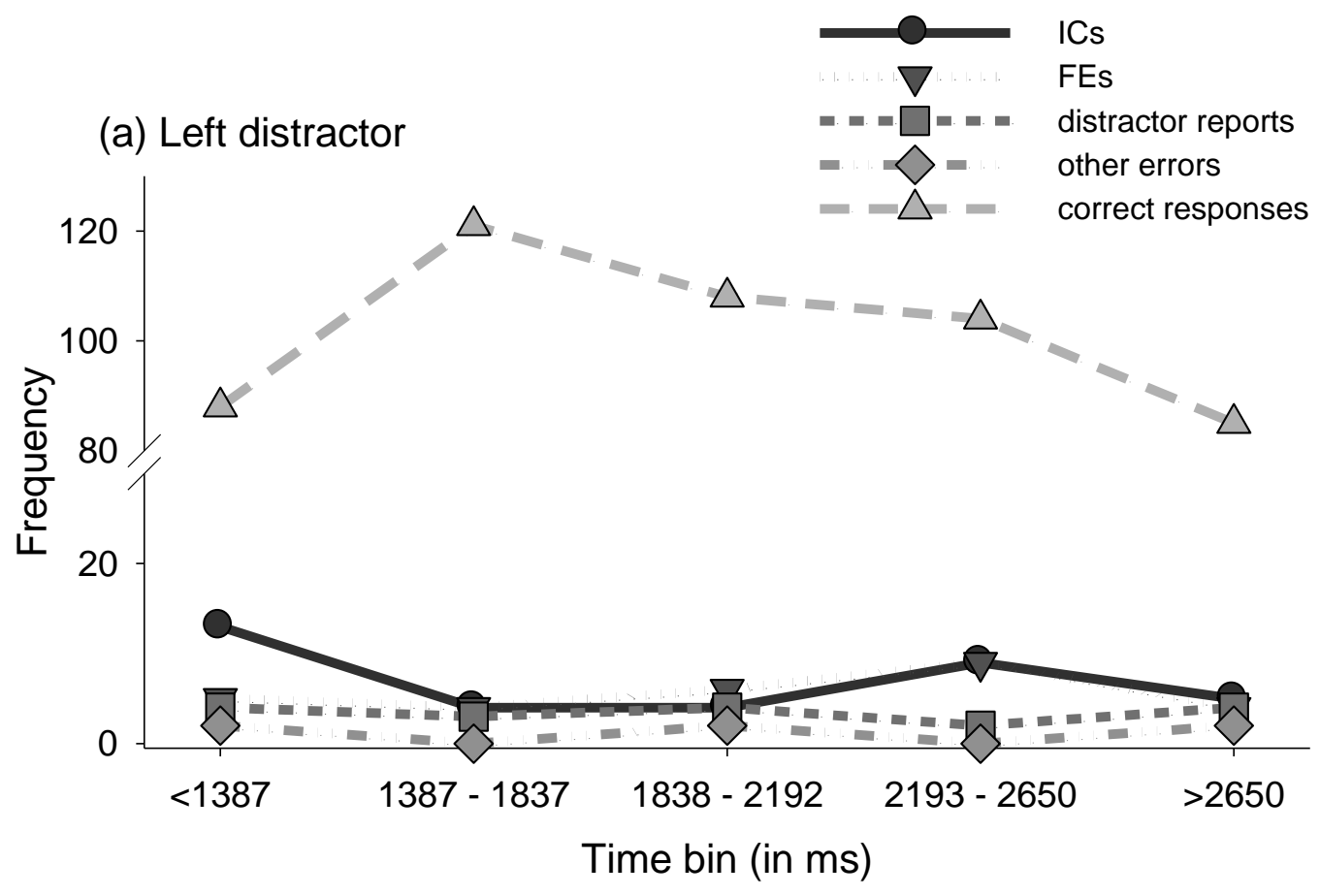

(b) Right distractor

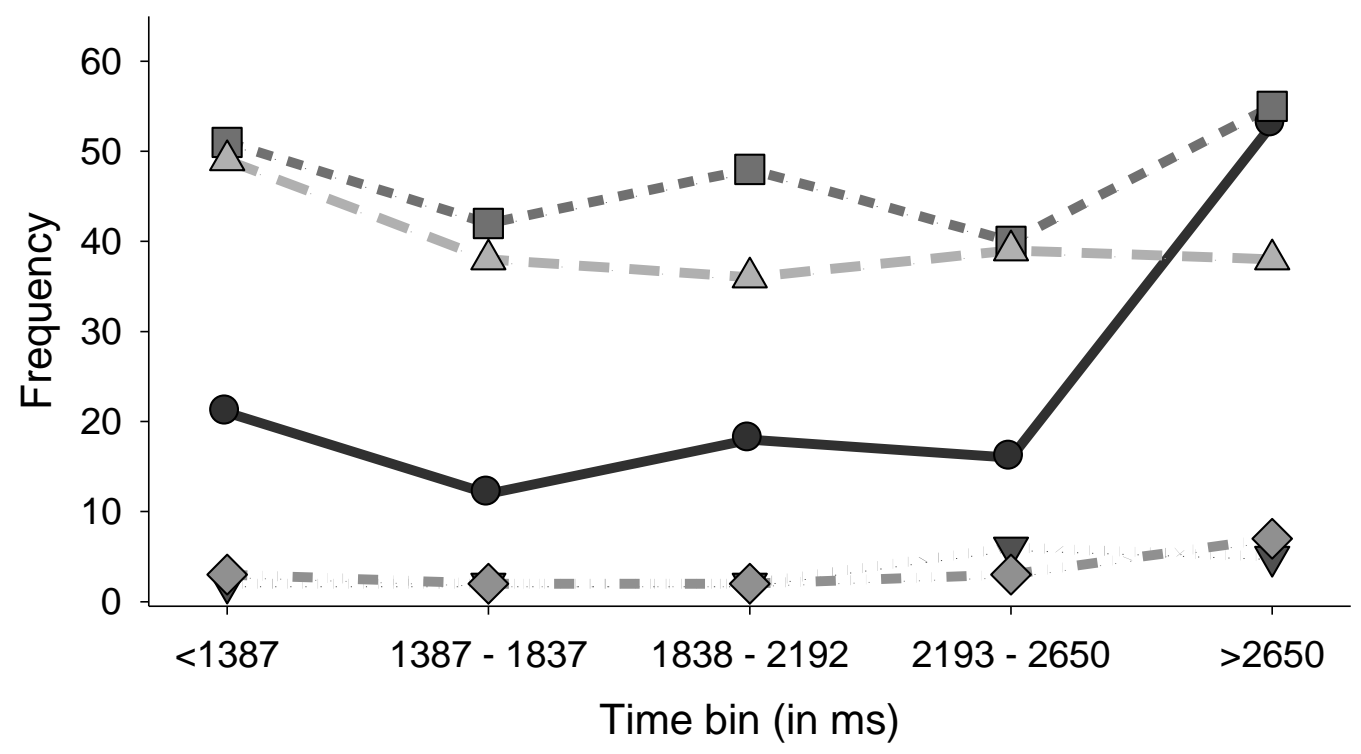

Figure 2. $\quad$ Experiment 1: The distribution of the correct responses and the four different error types (ICs, FEs, distractor reports, other errors) in the five time bins, $(a)$ for trials with a left field distractor, and (b) for trials with a right field distractor. 


\section{(a) Left distractor}

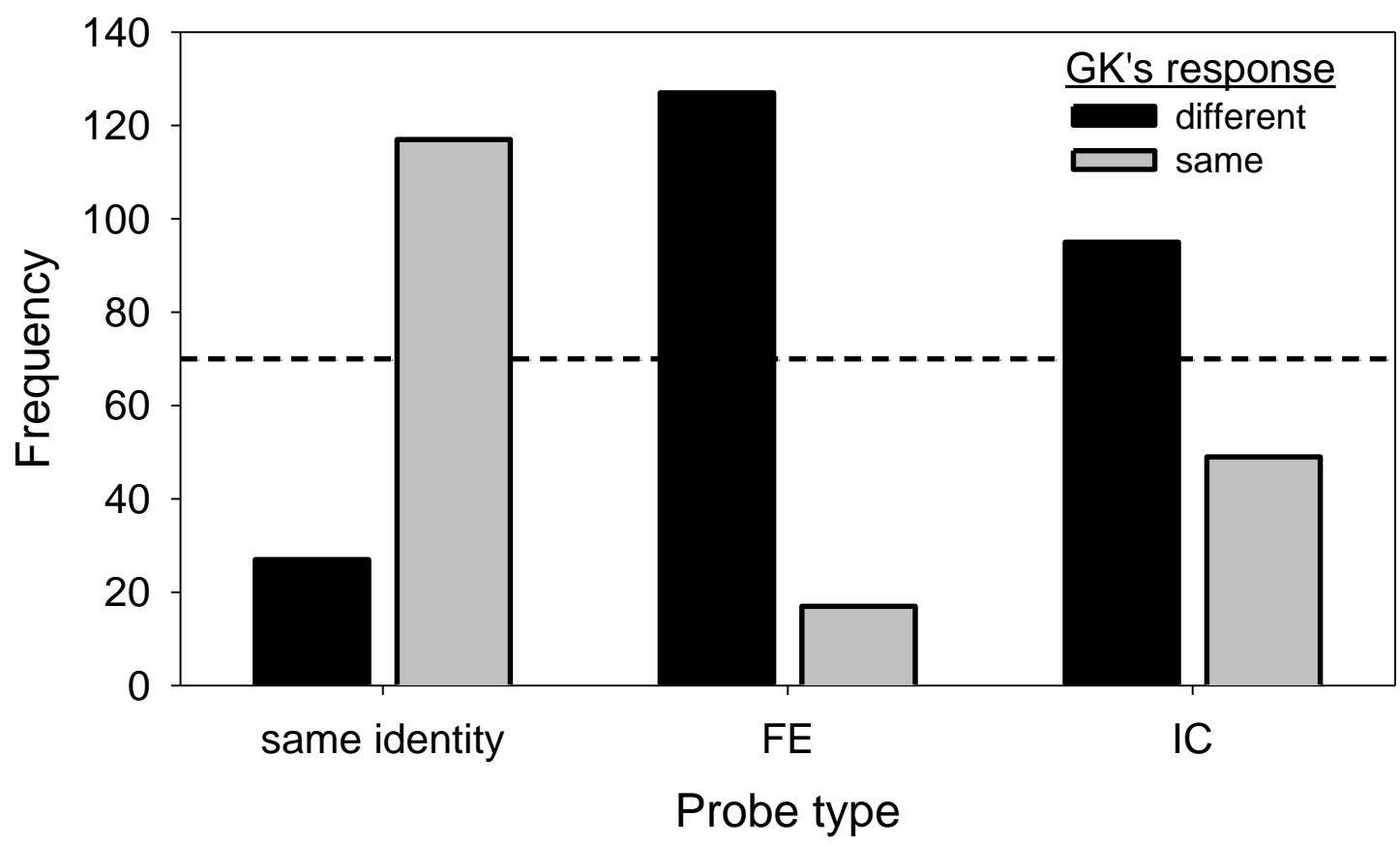

(b) Right distractor

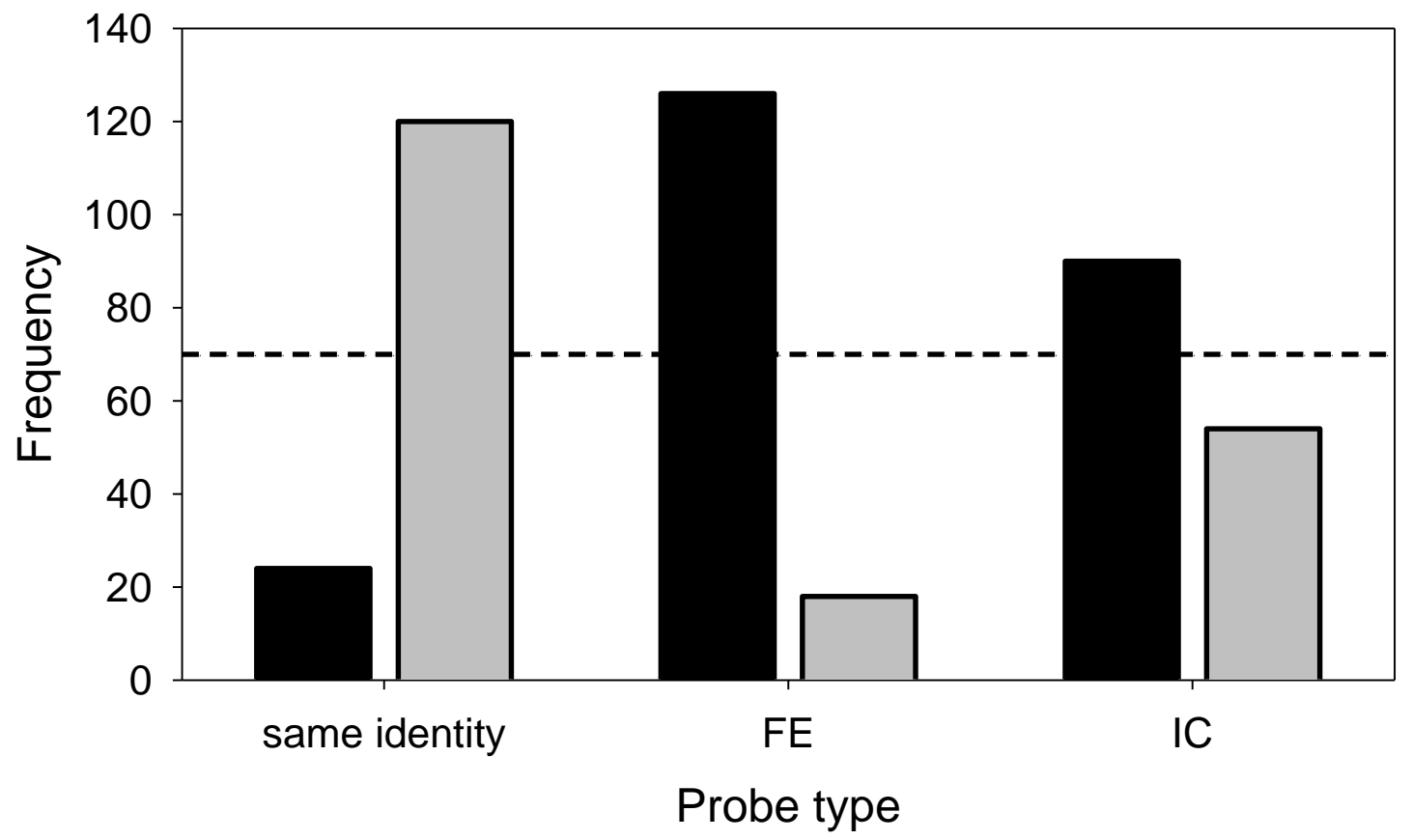

Figure 3. $\quad$ Experiment 2: The frequency of 'same' and 'different' answers in response to same identity, FE and IC probes, $(a)$ for trials with a distractor on the left, and $(b)$ for trials with a distractor on the right. The dashed line reflects chance. Correct answers are 'same' responses to same identity probes, and 'different' responses to FE and IC probes. Incorrect answers are 'different' responses to same identity probes (false negatives) and 'same' responses to FE and IC probes (false positives). 


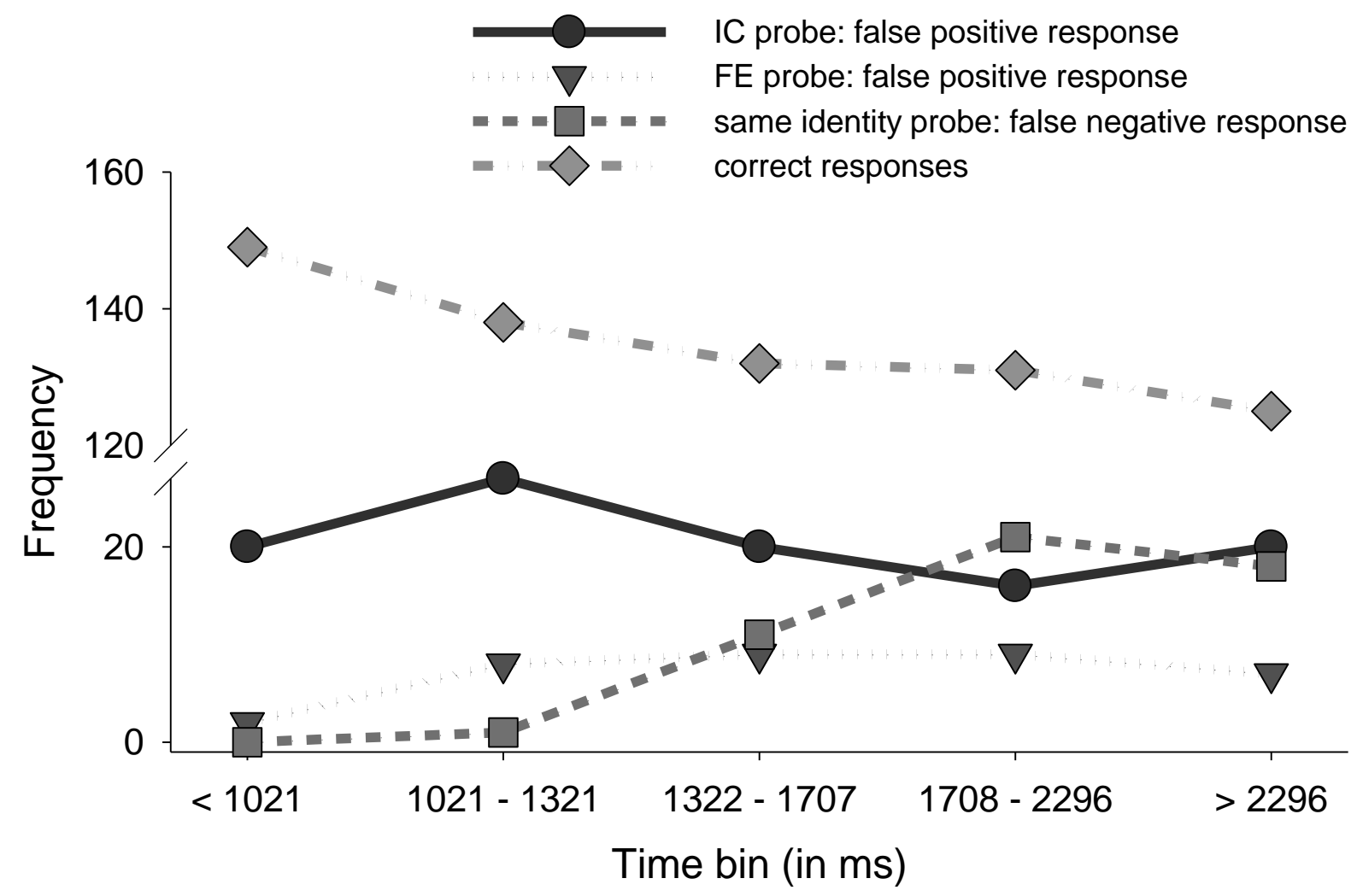

Figure 4. $\quad$ Experiment 2: The distribution of correct responses and the three different error types (ICs: false positives to IC probes, FEs: false positives to FE probes, false negatives to same identity probes) in the five time bins. 
(a) IC probes

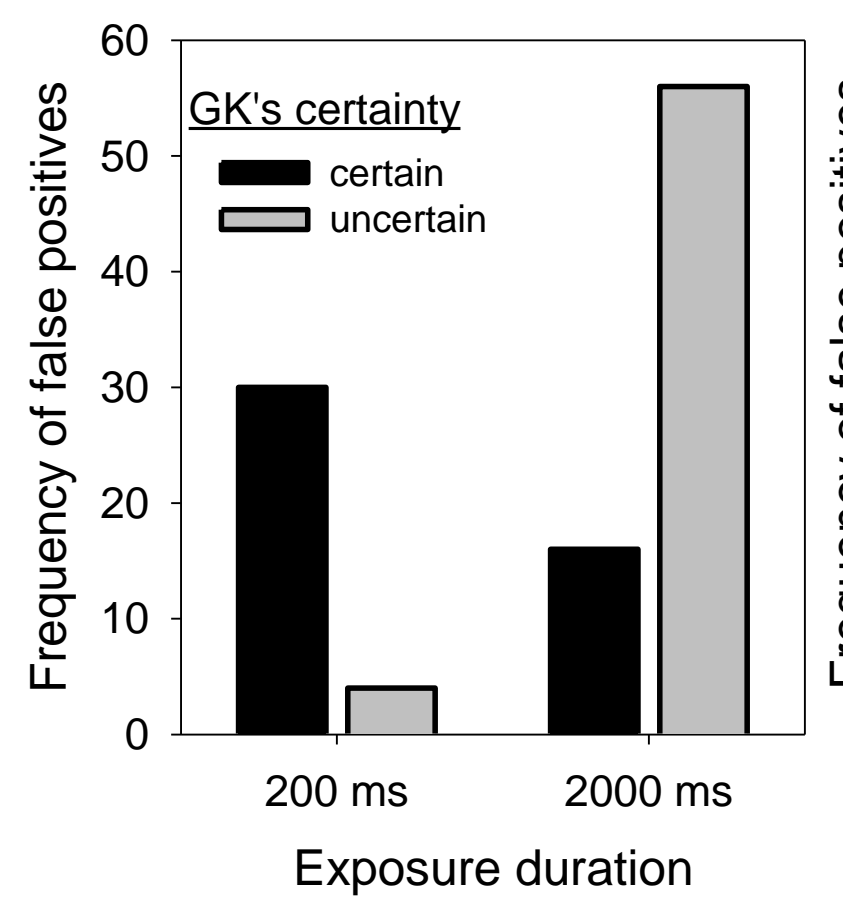

(b) FE probes

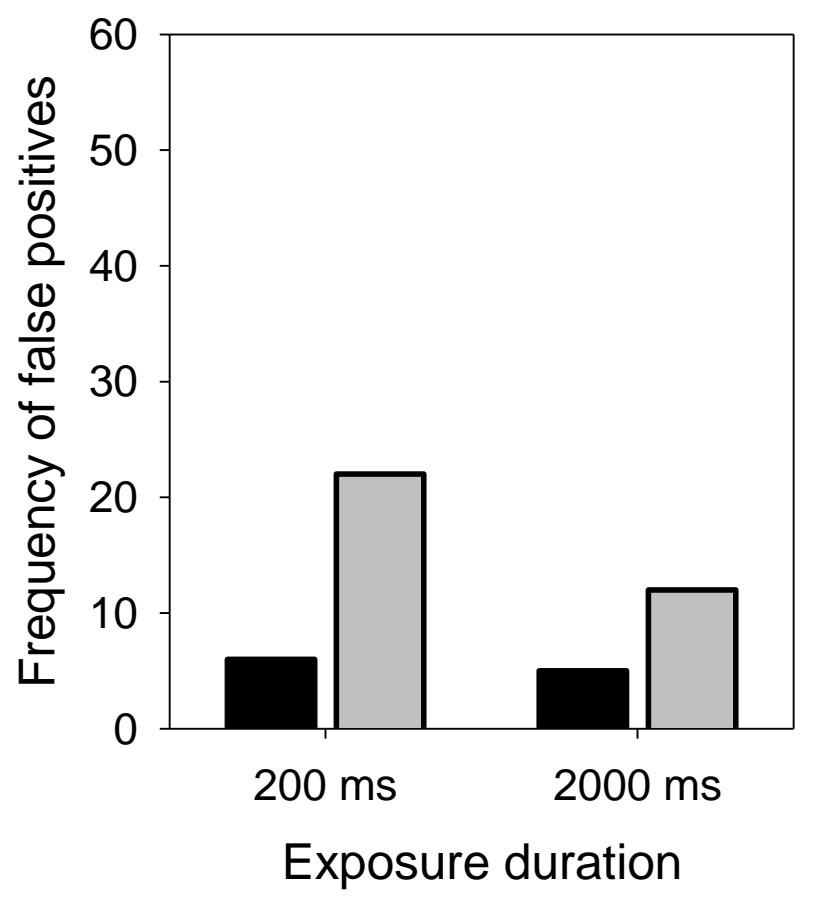

Figure 5. $\quad$ Experiment 3: The frequency of false positive answers in response to IC probes $(a)$ and FE probes $(b)$, coloured according to GK's certainty about his answer. 


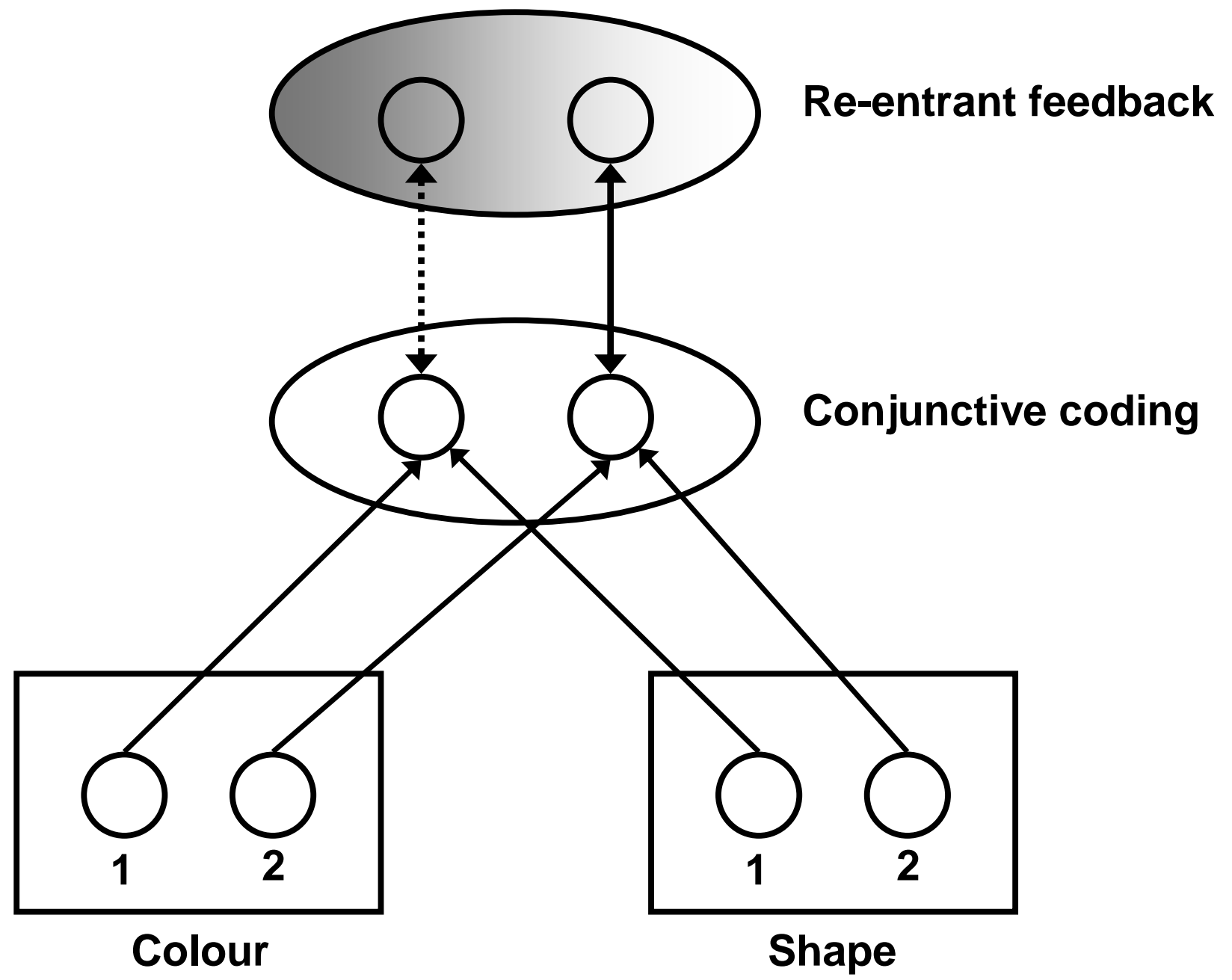

Figure 6. A re-entrant framework for conjunctive coding. Separate input modules code colours and shapes at two different locations ( 1 and 2 ), and feed activation forward to conjunctive coding units that form initial feature bindings. These initial feature bindings are stabilised by re-entrant feedback of the initially activated representation. We assume that GK has impairments at both the first (feed-forward) stage of binding and at the later (feed-back) stage. We also propose that the feedback stage was spatially biased to 'weight' activation from items in the right visual field, illustrated here by the grey-level gradient and the dotted feed-back line from the left-side units. Though we propose here local units for conjunctive coding, we note that conjunctive coding could take place through distributed representation over coarsely coded units. 